\title{
\begin{tabular}{l|l} 
Mibraries & DSpace@MIT
\end{tabular}
}

\author{
MIT Open Access Articles
}

\section{A concept for seeing-limited near-IR spectroscopy on the Giant Magellan Telescope}

The MIT Faculty has made this article openly available. Please share how this access benefits you. Your story matters.

Citation: Simcoe, Robert A., Gábor Fürész, Mark Egan, Timothy Hellickson, and Andrew Malonis. "A Concept for Seeing-Limited Near-IR Spectroscopy on the Giant Magellan Telescope." Edited by Christopher J. Evans, Luc Simard, and Hideki Takami. Ground-Based and Airborne Instrumentation for Astronomy VI (August 9, 2016).

As Published: http://dx.doi.org/10.1117/12.2234483

Publisher: SPIE

Persistent URL: http://hdl.handle.net/1721.1/108533

Version: Final published version: final published article, as it appeared in a journal, conference proceedings, or other formally published context

Terms of Use: Article is made available in accordance with the publisher's policy and may be subject to US copyright law. Please refer to the publisher's site for terms of use. 


\title{
A Concept for Seeing-Limited Near-IR Spectroscopy on the Giant Magellan Telescope
}

\author{
Robert A. Simcoe ${ }^{\mathrm{a}, \mathrm{b}}$, Gábor Fürész ${ }^{\mathrm{b}}$, Mark Egan $^{\mathrm{b}}$, Timothy Hellickson ${ }^{\mathrm{b}}$, and Andrew Malonis ${ }^{\mathrm{b}}$ \\ ${ }^{a}$ MIT Department of Physics, 77 Massachusetts Ave., Cambridge, MA 02139, USA \\ bMIT-Kavli Institute for Astrophysics, 77 Massachusetts Ave., Cambridge, MA, USA
}

\begin{abstract}
We present a simple seeing-limited IR spectrometer design for the Giant Magellan Telescope, with continuous $R=6000$ coverage from $0.87-2.50$ microns for a $0.7^{\prime \prime}$ slit. The instrument's design is based on an asymmetric white pupil echelle layout, with dichroics splitting the optical train into $y J, H$, and $K$ channels after the pupil transfer mirror. A separate low-dispersion mode offers single-object $R \sim 850$ spectra which also cover the full NIR bandpass in each exposure. Catalog gratings and H2RG detectors are used to minimize cost, and only two cryogenic rotary mechanisms are employed, reducing mechanical complexity. The instrument dewar occupies an envelope of $1.8 \times 1.5 \times 1.2$ meters, satisfying mass and volume requirements for GMT with comfortable margin. We estimate the system throughput at $\sim 35 \%$ including losses from the atmosphere, telescope, and instrument (i.e. all coatings, gratings, and sensors). This optical efficiency is comparable to the FIRE spectrograph on Magellan, and we have specified and designed fast cameras so the GMT instrument will have an almost identical pixel scale as FIRE. On the 6.5 meter Magellan telescopes, FIRE is read-noise limited in the $y$ and $J$ bands, similar to other existing near-IR spectrometers and also to JWST/NIRSPEC. GMT's twelve-fold increase in collecting area will therefore offer gains in signal-to-noise per exposure that exceed those of moderate resolution optical instruments, which are already sky-noise limited on today's telescopes. Such an instrument would allow GMT to pursue key early science programs on the Epoch of Reionization, galaxy formation, transient astronomy, and obscured star formation environments prior to commissioning of its adaptive optics system. This design study demonstrates the feasibility of developing relatively affordable spectrometers at the ELT scale, in response to the pressures of joint funding for these telescopes and their associated instrument suites.
\end{abstract}

Keywords: ELT, Near-Infrared, Spectrographs, echelle, white-pupil, seeing-limited (spectroscopy), GMT

\section{INTRODUCTION}

Many top-level scientific objectives for the Giant Magellan Telescope (GMT) require near-infrared (NIR) spectroscopy, but this capability has not been designed into the early operations architecture of the observatory. GMT's initial instrument suite consists of a seeing-limited multi-object optical spectrometer $\left(\right.$ GMACS $\left.^{1}\right)$ and a high-resolution fiber-fed optical spectrometer suitable for precision radial velocity measurements (G-CLEF ${ }^{2}$ ). Still, background-limited IR spectroscopy is a compelling application for Extremely Large Telescopes (ELTs) even in the seeing limit. This is in part driven by the early science these telescopes are expected to pursue regarding the early universe, but also because read noise limits the performance of IR spectrometers on existing telescopes (i.e. $D=6-10$ meters) with sufficient spectral resolution to split the OH-line foreground of the night sky. ${ }^{3}$ Even the James Webb Space Telescope (JWST) falls into this class - since JWST/NIRSPEC employs identical CMOS sensors on a 6.5 meter effective aperture, it will also be read-noise limited at wavelegnths where thermal emissivity does not dominate.

In time the demand for IR spectroscopy on GMT will be addressed by a followup generation of Adaptive Optics (AO) instruments, which includes an $R=50,000$ spectrometer operating at $\lambda=1.12-5.0 \mu \mathrm{m}$ for bright targets $\left(\right.$ GMTNIRS $^{4}$ ) and an $R=5000$ NIR fiber-fed integral field spectrometer with spatial sampling of $0.008^{\prime \prime}$ $(\min )$ to $0.050^{\prime \prime}(\max )$ in a single NIR band $\left(\right.$ GMTIFS $\left.^{5}\right)$. Early operation plans feature a staged commissioning sequence in which the telescope will initially be operated in the seeing limit during installation of the mount and

Further author information: (Send correspondence to R. Simcoe)

Ground-based and Airborne Instrumentation for Astronomy VI, edited by Christopher J. Evans, Luc Simard, Hideki Takami Proc. of SPIE Vol. 9908, 9908AA · C 2016 SPIE · CCC code: 0277-786X/16/\$18 · doi: 10.1117/12.2234483 
primary mirror segments. The AO system will then be commissioned in a subsequent phase as the enclosure and mount are completed and the primary mirror is properly phased.

Because GMT's baseline NIR spectrometers all require a fully-operational AO system, there is the potential for the AO commissioning schedule to drive the timescale on which GMT can pursue key science involving the high redshift universe, low mass stars, dust-obscured environments and broadband transients. We are therefore motivated to explore options for seeing-limited NIR spectroscopy at GMT, to better position the observatory to capitalize on these science opportunities during early commissioning.

This contribution presents the conceptual design of an affordable near-IR spectrometer developed in the context of an ongoing feasibility and trade study for the GMT Science Advisory Council (SAC). Our design is informed by past work on the FIRE spectrometer in operation at Magellan, ${ }^{6}$ which shares many science and technical requirements, but differs significantly in its implementation.

Like FIRE, this design (provisionally named SuperFIRE) is a single object cryogenic spectrograph with $R=6000$ and simultaneous coverage of the $y, J, H$, and $K$ bands in a single exposure. We have incorporated lessons learned from FIRE's construction and operational performance in SuperFIRE's specification. All gratings, optics, and detectors are available today as off-the-shelf procurements, with a goal of minimizing cost and schedule risk.

This relatively small instrument is easily accommodated in the volume envelope of GMTs Gregorian Instrument Platform. At this location it can be addressed by a dichroic diagonal tertiary, which transmits UV/optical light to the direct Gregorian optical spectrometer (GMACS), achieving complete and simultaneous coverage of the full optical and near-IR bands. This conceptual design study demonstrates the potential for development of relatively affordable instruments at the ELT scale, in response to the pressures of joint funding for these telescopes and their associated instrument suites.

\section{SCIENTIFIC MOTIVATION FOR EARLY NIR SPECTROSCOPY WITH GMT}

The GMT SAC has developed a representative program of science drivers for the observatory; ${ }^{7}$ these programs inform systems engineering requirements and therefore drive technical design of the telescope and instruments. Two of the seven major fields identified in this science book can only be pursued with IR instruments, and at least two additional fields are significantly enhanced by a NIR spectroscopic capability. We present here an incomplete but representative sampling of early science that would be enabled by access to a seeing-limited NIR spectrograph.

\subsection{First Light and Reionization}

Faint sources from the Epoch of Reionization (EoR) exhibit no flux at optical wavelengths (i.e. $\lambda \leq 9000 \AA$ in the observed frame), so NIR spectroscopy is required for GMT to pursue EoR science. This partially reflects the high redshift of the source population, but it also signifies absorption from intergalactic neutral hydrogen, which makes the universe act as a long-pass filter transmitting only IR photons to Earth from primordial galaxies. Because the most recent Planck measurements of the electron optical depth to the CMB suggest a low reionization redshift, ${ }^{8}$ there is renewed optimism that EoR studies using the $\mathrm{CMB}$, redshifted $21 \mathrm{~cm}$ radio emission, ${ }^{9}$ and GMT will soon converge in redshift. ${ }^{10}$

Two principal observational strategies are used to characterize the EoR: spectroscopy of the energetic sources which ionize the surrounding intergalactic medium (IGM) ${ }^{11-14}$ and direct characterization of the evolving baryonic matter field (i.e. the IGM itself). ${ }^{15,16}$ JWST will excel in deep surveys of the primordial galaxy population, and will be especially powerful at gathering redshifts and measuring rest-frame optical emission lines in the $L$ and $M$ bands where thermal emission from the Earth's atmosphere limits deep observations from the ground.

Since the collecting area of JWST is similar to today's mid-sized ground-based telescopes, sensor read noise will limit rest-frame UV observations of these galaxies with NIRSPEC except at low spectral resolution. JWST should therefore lead the way in identifying these galaxies and measuring redshifts, but the increased collecting area of ELTs is needed to deliver the higher spectral resolution that allows the study of astrophysical velocity structure and identification of weaker diagnostic UV lines above the read noise floor. The velocity structure 
of Ly $\alpha$ lines is of particular interest for understanding radiative transfer as the absorption topology changes around these objects, and additional metal line transitions of differing ionization potential can be used to study metallicity and diagnostics of the radiation field. The He II $1640 \AA$ line will be of particular interest, because it can be identified even in very low metallicity environments unpolluted by early stars. This weak transition falls in the $J$ and $H$ bands for EoR galaxies, where GMT's collecting area coupled with high NIR spectral resolution should bring out lines of low equivalent width.

Observations of high-redshift EoR quasars can be used to characterize the damping wing in neutral hydrogen near the QSO emission redshift, ${ }^{17,18}$ and to survey foreground heavy-element absorbers. ${ }^{19}$ Such observations require spectral resolution of $\sim 50 \mathrm{~km} / \mathrm{s}$ to identify important doublets such as CIV and MgII.

\subsection{Astrophysical Transients}

Studies of explosive transients benefit from rapid response/setup time and the broadest possible wavelength coverage. Althoguh GMT's optical spectrographs will be able to capture transients, an optimal instrument configuration would observe at low-to-moderate spectral resolution from the ultraviolet to the NIR simultaneously.

The dynamic sky is largely unexplored at IR wavelengths but there are indications on both the theoretical and experimental fronts suggesting that this may be a fruitful growth area. Recent Spitzer-based time-domain surveys of nearby galaxies have revealed many transients that are only visiable in the IR; ${ }^{20}$ these may represent stellar mergers in obscured environments. ${ }^{21}$ Althoguh still uncertain, early predictions of the "kilonova" afterglow lightcurves for binary neutron-star mergers (as would be seen by LIGO ${ }^{22,23}$ ) suggest high opacity from Lanthanide elements that would yield an SED peak in the NIR. ${ }^{24}$ Further in the future, spectroscopy of high-redshift gammaray bursts could improve constraints on the IGM neutral fraction.

The primary requirements for transient science are rapid availability, spectral coverage, and sensitivity. A significant fraction of GMT observing time is likely to be in queue mode (enabling rapid response for followup) and the large collecting area will maximize sensitivity. Configuration of a NIR spectrograph fed by a dichroic split would allow UV-throguh- $K$ observations of transients, maximizing the information content of each targetof-opportunity observation. Because GMT is co-located in Chile with LSST and will thus have access to a rich array of transients, this connection should be especially strong during GMT's early operations.

\subsection{Galaxy Assembly and Evolution}

Deep imaging campaigns with HST in recent years have revealed a surprising growth in the size of early type galaxies from $z \sim 2$ to the present, ${ }^{25,26}$ as well as a population of compact blue galaxies that may be early ancestors of elliptical galaxies. Deep campaigns are underway to study the velocity dispersions and stellar populations of these systems using IR spectroscopy (which probes the rest-frame optical at these redshifts). These investigations require full-night integrations per object, or fortuitous alignment of gravitational lenses on current telescopes, but ELTs should facilitate statistical studies of this population. With samples of these objects it will be possible to study the buildup of the fundamental plane and mass-metallicity relation in more depth than has been achieved in current pathfinder surveys. ${ }^{27}$

It will be particularly powerful if SuperFIRE can observe in parallel with GMT's optical spectrometer in similar fashion as X-Shooter. ${ }^{28}$ This will enable spectroscopy of star forming galaxies from their rest-frame UV through optical. With the higher resolution and continuum sensitivity afforded by GMT, it will be possible to resolve velocity structure in both galactic outflows and inflows, while establishing a precise measure of the systemic rest frame via optical emission lines. Key design drivers in this space include velocity resolution of $\sim 50 \mathrm{~km} / \mathrm{s}$ (i.e. below the velocity dispersion of dark matter halos at $z \sim 2$ ), broad wavelength coverage, and sky-limited continuum sensitivity for stellar population studies.

\section{DERIVED INSTRUMENT REQUIREMENTS}

The basic design parameters for SuperFIRE are listed in Table 1. They are derived both from the demands of the target science described above, and from experience operating the FIRE spectrometer since 2010 at Las Campanas. The rationale for each specification may be summarized briefly as follows: 
Table 1. Key Science-Driven Design Requirements for SuperFIRE

\begin{tabular}{|l|l|l|l|l|}
\hline & Requirement & Goal & Driver & SuperFIRE \\
\hline \hline Slit Width & $0.7^{\prime \prime}$ & $0.7^{\prime \prime}$ & LCO Seeing/throughput & $0.7^{\prime \prime}$ \\
\hline Slit Length & $7.0^{\prime \prime}$ & $10.0^{\prime \prime}$ & Sky model estimate & $8.0^{\prime \prime}$ \\
\hline Pixel Pitch & $0.10^{\prime \prime}$ & $0.12-0.15^{\prime \prime}$ & Sky-limited noise & $0.12(y, J), 0.10(H, K)$ \\
\hline Spectral $R$ & 4500 & 6000 & OH-free spectral path & 6000 \\
\hline Low- $R$ mode & none & $R \sim 1000,1^{\prime}$ longslit & Transients, low-M stars & $R=800,15^{\prime \prime}$ slit \\
\hline
\end{tabular}

- Slit width: SuperFIRE should deliver its target resolution at a slit width matched to Las Campanas' natural seeing for maximum throughput. FIRE's nominal slit width is $0.6^{\prime \prime}$ since a survey of the site characteristics measured this median value. Since commissioning we have measured slightly higher values at typical airmass, with correspondingly lower throughput. To compensate for this effect we widen SuperFIRE's slit slightly to $0.7^{\prime \prime}$

- Slit Length: This requirement is informed by experience observing with FIRE's $6.0^{\prime \prime}$ slit, which provides adequate off-object sampling to model and remove the background sky. However we aim to increase this length closer to $10^{\prime \prime}$ to prevent extended objects from approaching the slit boundaries, and to provide more path for larger dithers along the slit extent. This places demands on the cross dispersing elements which must deliver adequate order separation at this increased slit length.

- Spectral resolution: This requirement has several drivers. First, we wish to maximze the amount of spectral path free of contamination from terrestrial $\mathrm{OH}$ emission (which argues for high resolutions ${ }^{3}$ ) while also keeping the instrument in the background-limited noise regime. Second, we wish to offer a higher$R$ alternative to JWST that takes advantage of GMT's collecting area to enable unique science during early operations. At $R=6000$, SuperFIRE would have velocity resolution of $\Delta v=50 \mathrm{~km} / \mathrm{s}$, suitable for resolving multiplet lines, studying kinematic velocity struture in diverse sources, and performing basic chemical abundance work.

- Pixel Pitch: Early observations with FIRE revealed that the broadband sky background betwen $\mathrm{OH}$ emission features is darker than had been anticipated, such that the $y$ and $J$ band spectral regions are largely read noise limited on Magellan. ${ }^{3}$ Because IR spectrographs on 6-10 meter telescopes suffer from read noise, the scale-up to GMT's larger aperture should produce large gains in sensitivity even when seeing limited. Assuming identical sensor noise, this requires that the plate scale in arcseconds per pixel is matched between FIRE and SuperFIRE so that increase in aperture is not offset by diffusion of photon flux. FIRE employs a single H2RG sensor with sampling of between $0.09-0.15^{\prime \prime}$ per $18 \mu \mathrm{m}$ pixel (with variable sampling from anamorphism of the prism cross-disperser). For SuperFIRE to operate in the sky-limited regime, we have specified sampling of $0.12-0.15^{\prime \prime}$ per pixel, with special emphasis on coarse sampling in the $y J$ band. In $K$ and (to a lesser degree) $H$, the background is dominated by thermal emission. Here coarse sampling is less critical so we relax the sampling requirement in the redder bands to $0.10^{\prime \prime}$ per pixel.

- Wavelength Range: Much like FIRE and X-Shooter, SuperFIRE is designed to deliver single-shot spectra of the full NIR band. Its blue wavelength requirement is set by the desire to cover Ly $\alpha$ for EoR sources at $z \geq 6.5$ with a goal of $z \geq 5.5$. In practice the red optical bands may get split by a dichroic at the $z / y$ boundary, with blue light being transmitted to the facilty optical spectrometers*. Continuous wavelength coverage is critical for galaxy and absorption line surveys where the redshifts of interesting sources are not typically known a priori, and simultaneous coverage of multiple lines (i.e. in identical weather conditions) is optimal for calcualtion of physical diagnostic ratios.

${ }^{*}$ Although dichroics are typically used in a configuration which reflects blue light, in this case we are constrained to use in the opposite configuration (red reflecting) because GMACS can only be mounted at the Direct Gregorian focus. 
Table 2. Key Programmatic Design Requirements for SuperFIRE

\begin{tabular}{|l|l|l|l|l|}
\hline & Requirement & Goal & Driver & Baseline \\
\hline \hline Echelle Grating & COTS & RGL Catalog & Cost/Schedule & RGL Catalog \\
\hline Sensor & COTS & H2RG & Cost $/$ pixel pitch & $3 \times$ H2RG \\
\hline Volume Envelope & GMT Instrument ICD & & Cost & $1.2 \times 1.5 \times 1.8 \mathrm{~m}$ \\
\hline Cooling Method & LN2 & LN2 & Low vibration (reqt) & Dual LN2 reservoirs \\
\hline Mass Budget & $\leq 7000 \mathrm{~kg}$ & $\leq 2000 \mathrm{~kg}$ & GMT reqt. & $1350 \mathrm{~kg}$ \\
\hline
\end{tabular}

The most difficult technical challenge is imposed by the pixel scale requirement, which is fundamental if SuperFIRE is to realize the full advantage of installation on an ELT-scale telescope. Because of GMT's four-fold increase in diameter relative to Magellan, the spectrograph camera must be much faster to achieve the same pixel scale. FIRE's camera has a monochromatic focal ratio of $\mathrm{F} / 3.5$, but its entrance pupil is nearly twice as large as this would imply because of chromatic spread after the echelle grating and cross disperser.

This configuration is difficult to execute at GMT's scale, because of technical risk for cameras faster than F/1.0. Our approach has been to design around the H2RG sensor which has significant heritage, but also has the largest pixel size $(18 \mu \mathrm{m})$ among Teledyne's large format HgCdTe sensors - the H4RG is offered at 15 and $10 \mu \mathrm{m}$, so would require an F/0.8 or F/0.6 camera at the same plate scale. Even with H2RG sensors, SuperFIRE's coarse pixel scale requires cameras with monochomatic focal ratio nearing F/1.0. This drove us to a white pupil configuration $^{29}$ for the remainder of the spectrograph, to contain further growth of the camera aperture caused by displacement from a polychromatic pupil.

\section{OPTICAL DESIGN}

\subsection{Overview and Comparison with Heritage Designs from Magellan/FIRE}

SuperFIRE's optical layout (Figure 1) utilizes a compact white-pupil configuration with a catalog R2 echelle grating from the Newport/Richardson Lab. After dispersion from the Echelle grating and reflection from the pupil transfer mirrors, the beam is split by dichroics into three channels. These arms are serviced by individual VPH cross dispersers and H2RG sensors which cover $870-1400 \mathrm{~nm}(y J), 1400-1800 \mathrm{~nm}(H)$, and $1800-2500$ $\mathrm{nm}(K)$, respectively. The beam diameter at the white pupil is $120 \mathrm{~mm}$, and is matched to the camera's entrance pupil.

Although SuperFIRE's fundamental design parameters (Table 1) are almost identical to those of Magellan/FIRE, the specific implementation outlined above is entirely different because of particular demands of scaling such an instrument to the ELT class. Moreover we have made every effort to minimize cost and complexity, by using COTS components for all of the major technical systems, and by driving the cold instrument volume as small as possible.

For example, the defining feature of FIRE's design is a network of Infrasil and ZnSe prisms used for crossdispersion. These prisms maintain high throughput over FIRE's full factor of $\sim 3$ in wavelength coverage using a single arm and detector. However procurement of the large-thickness ZnSe prisms was a schedule driving item for FIRE - they require large apex angles and multiple passes to achieve the angular dispersion needed for order separation in the NIR. In scaling from Magellan to GMT, the beam diameter at fixed $R$ increases by $4 \times$, which leads to an corresponding increase in prism thickness that makes this design difficult or even impossible.

Once FIRE's prism cross-dispersed architecture is discarded in favor of gratings, we must split SuperFIRE into multiple channels to maintain a high blaze efficiency across the broad instrument bandpass. Atmospheric absorption bands between $y J, H$, and $K$ provide convenient locations for these splits, and define relatively narrow windows where a high VPH efficiency can be maintained.

The switch from prism to grating cross dispersers (which can achieve much higher dispersion) affords increased flexibility in the choice of echelle gratings. Because most prism materials have weak dispersion in the NIR, 


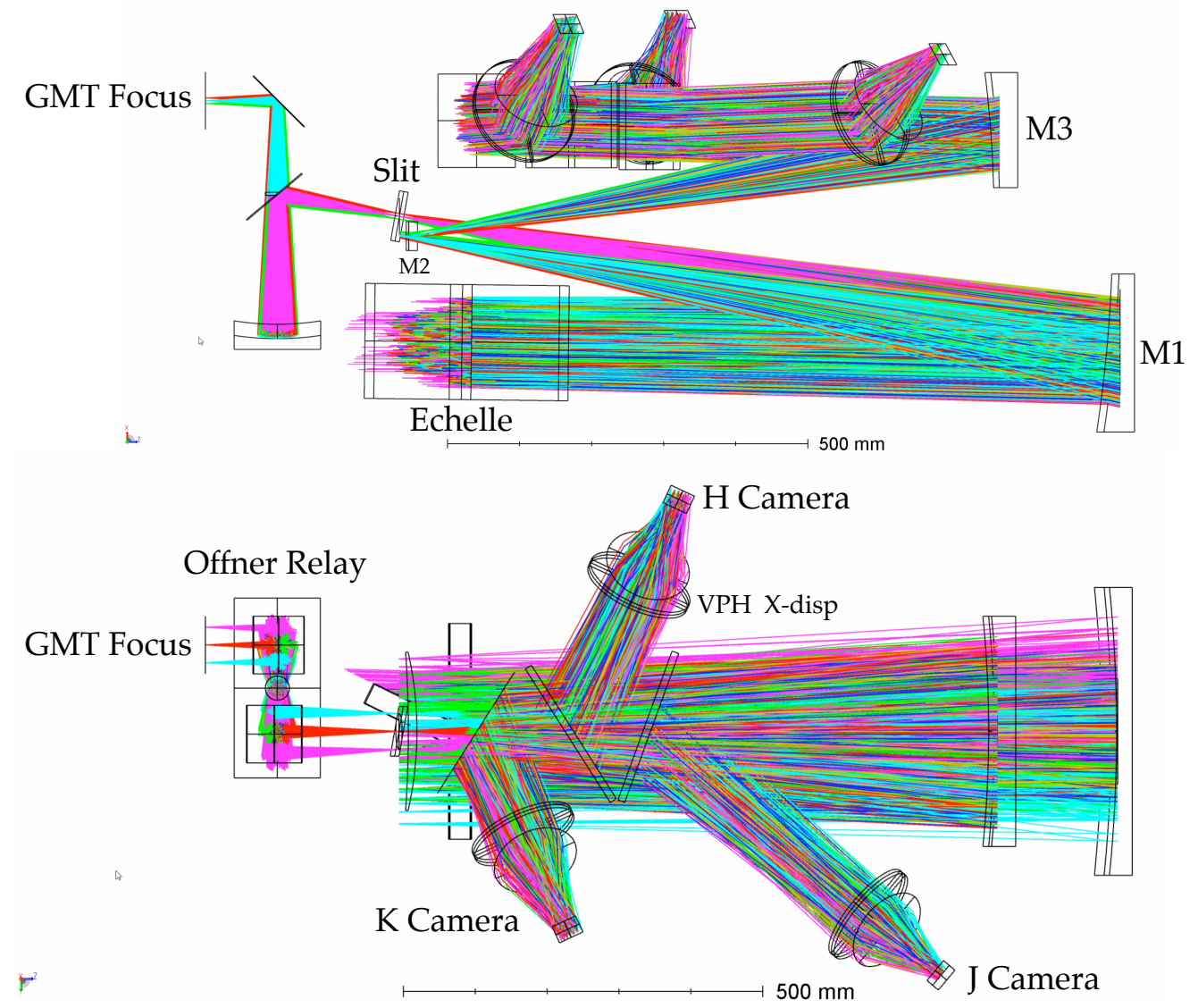

Figure 1. Basic layout of SuperFIRE as seen from the side (top panel) and from above (bottom panel). The spectrograph cameras are shown as paraxial surfaces for simplicity, and elements are labeled according to descriptions in the text. As seen in the side view, and optical bench splits the upper half of the instrument (which contains the cross-dispersers and cameras) from the bottom half (which contains the collimator and echelle). In the lower panel, both dispersion configurations are shown - the echelle configuration with tilted grating, and Low-dispersion mode where the grating is replaced by a flat.

instruments like FIRE operate best at R1 $\left(\theta_{\text {blaze }}=45^{\circ}\right)$ in Echellette mode, where one runs in low order numbers to achieve a wide free spectral range (and hence maximize order separation for a given dispersion). With SuperFIRE's grating cross-dispersers, we specify a coarser ruling at R2 $\left(\theta_{\text {blaze }}=63^{\circ}\right)$ at higher order number. This halves the size of the beam required to achieve a given resolution, so it is a substantial advantage when scaling up from Magellan to GMT-i.e. GMT+SuperFIRE is a factor of $\sim 2$ larger than Magellan+FIRE, not a factor of four.

Operation at higher order number also implies a smaller free spectral range than FIRE and hence more orders are needed to meet the requirement of coverage over the full bandpass. However because we have moved from one spectrograph arm in FIRE to three in SuperFIRE (driven by the swtich from prism to grating cross-dispersers) we already have three times as much detector area and can easily accommodate the increased number of orders.

\subsection{Pre-Slit Optics}

Before entering the spectrograph slit, light from the F/8 telescope beam is transferred through an Offner relay to baffle thermal backgrounds. The symmetric Offner will be constructed of gold-coated, diamond-turned and post-polished Aluminum. Its entrance and exit are aligned to the telescope and instrument respectively using two optical flats; these can be actuated to correct for flexure between the cold mass and the external GMT 

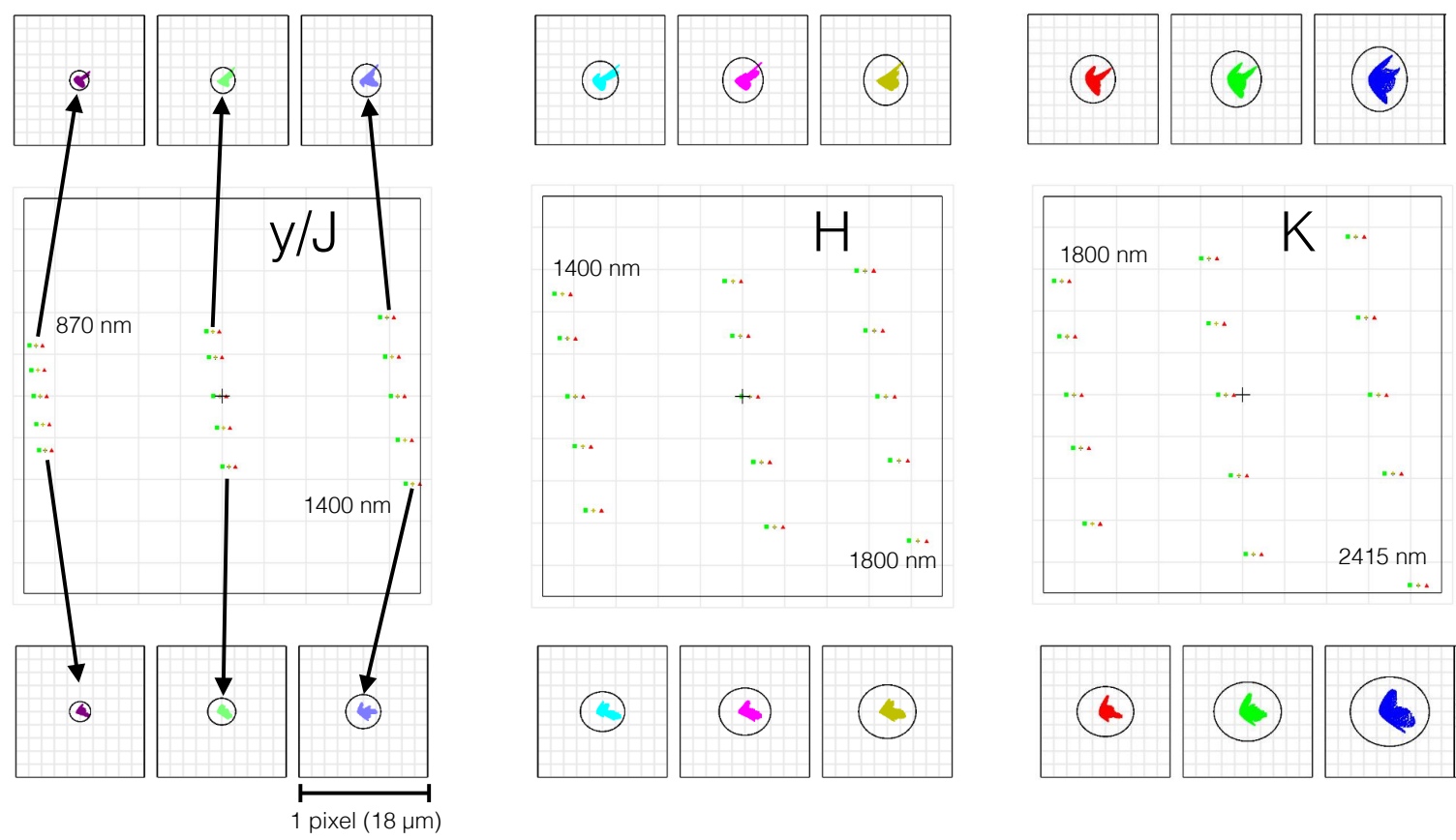

Figure 2. Footprint diagrams at the focal plane sensor for the three cameras. In each panel, we show five wavelengths across the free spectral range (top to bottom) for the bluest, central, and reddest orders from left to right, with wavelengths labeled. For each configuration of order number and wavelength, three field angles are shown corresponding to the center of the slit, and $\pm 4^{\prime \prime}$, i.e. the slit edges. Inset in each diagram are $1 \times 1$ pixel boxes $\left(0.122^{\prime \prime}\right.$ square $)$ indicating the image quality of the spectrograph with paraxial cameras (i.e. isolating aberrations from the spectrograph optics). The circles in these spot diagrams denote the diffraction limit, indicating that aberrations for the total system are dominated by the cameras.

guiders. This stage can also take up slight misalignments in the pointing center between SuperFIRE and optical spectrometers when they are used simultaneously.

The Offner's $60^{\prime \prime} \times 60^{\prime \prime}$ field is imaged onto a reflective slit plate, which is tilted slightly to accommodate an IR slit viewing camera (an engineering grade H2RG) for acquisition and slow guiding.

\subsection{White Pupil Layout and Paraxial Performance}

After the slit plane, the basic layout is that of an asymmetric white pupil echelle, as shown in Figure 1. The diverging beam is collimated via an F/8 off-axis paraboloid (M1) with 1-meter focal length, which projects a $132 \mathrm{~mm}$ diameter pupil onto an R2 Echelle grating (described below). The dispersed return beam is passed back from M1 to an intermediate focus just below the slit.

Light at the intermediate focus reflects off a convex $180 \times 40 \mathrm{~mm}$ toroidal Mangin mirror (M2) and is relayed to an concave ellipsoidal "pupil transfer" mirror (M3). The Mangin mirror is designed to remove Petzval curvature inherent to the white pupil relay at the intermediate focus, before the beam enters the camera. This is desirable because the downstream cross-dispersers break the rotational symmetry of the aberrations, so correcting prior to cross-dispersion eliminates the fabrication and alignment challenge of cylindrically figured lenses in the camera, close to the detector. This advantage multiplies three-fold for SuperFIRE's three channels, and also allows the camera optics to be aligned and tested independently, outside the context of the full spectrograph. This is particularly critical for fast cryogenic optics, and because of MIT's heritage with the FIRE and TESS projects we already have the critical equipment needed to perform full interferometric (double-pass) qualificaiton of the SuperFIRE lens assemblies at operating temperature. 
A small wedge is incorporated in the Mangin prescription to mitigate pupil ghosting. We have incorporated a slight asymmetry into the design by reducing the focal length of M3 (800 mm) relative to M1. This trade reduces the size of the collimated beam at the white pupil (and hence the cameras) from $132 \mathrm{~mm}$ to $120 \mathrm{~mm}$.

The dichroic split between SuperFIRE's three arms takes place between M3 and the white pupil, because we wished to locate the white pupil as close as possible to the cameras' entrance aperture. Each of the three arms has an independently ruled VPH cross disperser.

Figure 2 shows the performance of this layout with the cameras replaced by paraxial surfaces to isolate aberrations from the spectrograph. The three panels represent the three arms of SuperFIRE with the full H2RG foootprint shown at center. Primary dispersion runs from top to bottom in this view and cross dispersion from left to right. In each panel, three representative orders are displayed for each channel, over five wavelengths spanning the free spectral range; green/yellow/red symbols denote three field angles correpsonding to the center and extrema of the slit for each wavelength.

The insets in each panel represent a $1 \times 1$ pixel box, corresponding to $0.122^{\prime \prime}$ in $J$ and $0.10^{\prime \prime}$ in $H$ and $K$. Each inset contains a solid circle indicating the extent of the Airy disk, clearly indicating that the white pupil layout with Mangin mirror can deliver a diffraction limited wavefront to the spectrograph cameras.

\subsection{Grating Selection}

SuperFIRE's echelle grating is a catalog ruling available from the Newport/Richardson Grating Lab. As described above, it is blazed at $63^{\circ}$ for operation at $\mathrm{R} 2$ rather than FIRE's $\mathrm{R} 1$ to achieve $R=6000$ for a compartively small beam diameter (for an ELT) of $132 \mathrm{~mm}$. It operates in orders $m=24-66$ in a near-Littrow configuration. The order coverage is split unevenly between the three channels, with the $K$ band arm covering 8 orders $(m=24-31)$, the $H$ arm covering 9 orders $(m=32-40)$, and the $y J$ arm covering 25 orders $(m=41-65)$. This imbalance is driven by the convenience of placing the dichroic splits in atmospheric absorption bands, and the smaller FSR at higher order number. It has the undesirable consequence of limiting the slit length to a level below what the optics can accommodate, but for now this is outweighed by the desire not to place the dichroics in spectral regions important for EoR science.

Each channel incorporates an individually ruled VPH transmission grating that is roughly coincident with the white pupil, immediately in front of the camera. These gratings have ruling densities of 500, 480, and 330 lines/mm for the $J, H$, and $K$ arms, with preliminary gel thickness from $5-11 \mu \mathrm{m}$.

\subsection{Spectrograph Cameras}

Fast spectrograph cameras constitute the primary challenge in designing a sky-noise limited and seeing-limited IR spectrometer for ELTs. White pupil designs help by minimizing the camera's aperture for a given beam size, but the camera focal length is fixed by the respective plate scales of the telescope and sensor, so as the telescope's aperture grows the camera focal ratio must decrease in proportion to focus enough sky photons to exceed read noise, even for GMT.

Table 3. Designs for three channels

\begin{tabular}{|l|l|l|l|}
\hline & $y J$ Channel & $H$ Channel & $K$ Channel \\
\hline \hline$\lambda(\mathrm{nm})$ & $870-1400$ & $1400-1800$ & $1800-2500$ \\
\hline Orders $(m)$ & $41-65$ & $32-40$ & $24-31$ \\
\hline Plate scale $\left({ }^{\prime \prime} / \mathrm{pix}\right)$ & 0.122 & 0.100 & 0.100 \\
\hline Focal length $(\mathrm{mm})$ & 120 & 150 & 145 \\
\hline Focal ratio & $\mathrm{F} / 1.0$ & $\mathrm{~F} / 1.25$ & $\mathrm{~F} / 1.2$ \\
\hline FoV $\left(^{\circ}\right.$ diam $)$ & 18 & 15 & 16 \\
\hline
\end{tabular}




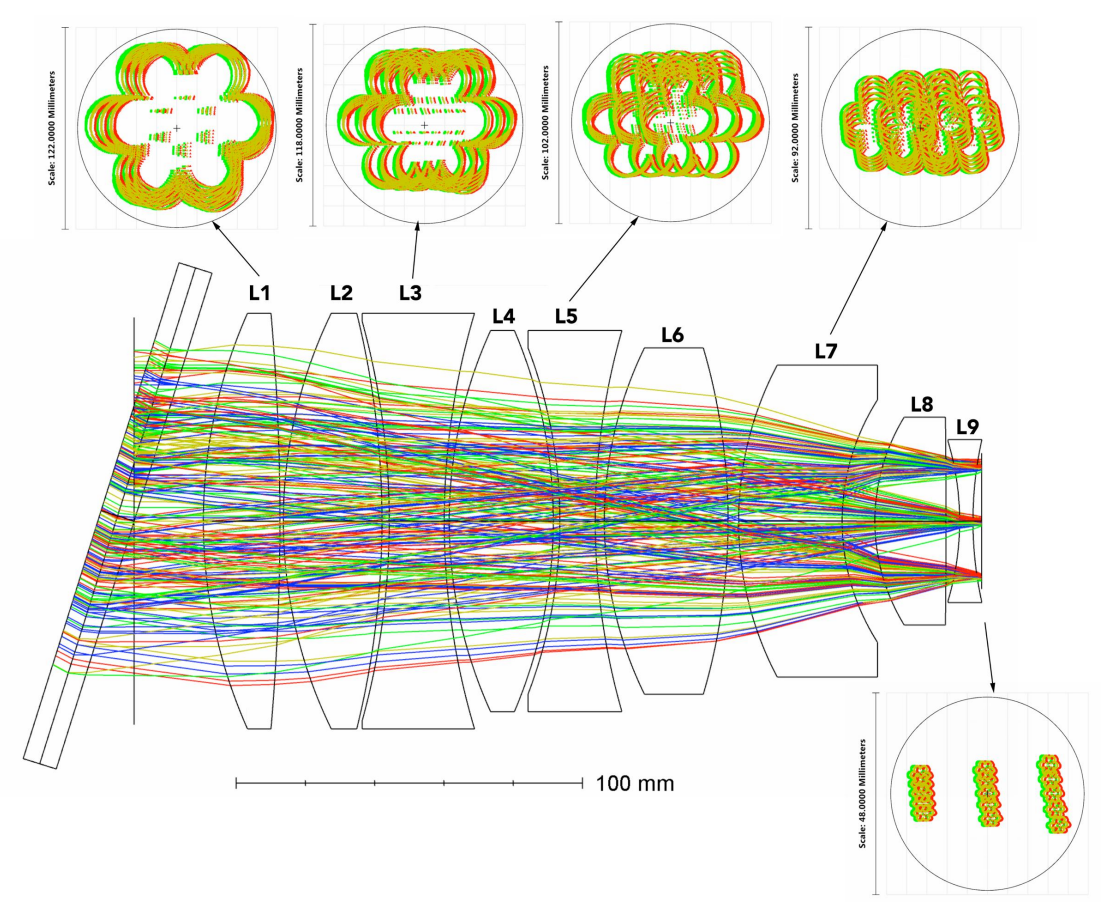

Figure 3. Preliminary layout of the F/1.0 J band spectrograph camera. This is a 9-element, all spherical, airspaced design to minimize CTE-induced stresses from bonded elements in the cryostat. The entrance pupil, located near the VPH grating (which is shown at left), is $120 \mathrm{~mm}$ in diameter. This is the fastest camera in the instrument. Footprint diagrams are shown for several representative lenses in the optical train; the small overlapping foorptint for all field angle/wavelength combinations reveals the motivation for using a white pupil configuration. This layout is only a conceptual design and will be further optimized through better glass selection and use of aspheres.

We have carefully considered this trade for SuperFIRE, which employs three separate cameras for its three channels. The vital parameters of these cameras are listed in Table 3. GMT has a native plate scale of roughly $1^{\prime \prime} / \mathrm{mm}$, which must be reimaged to $1^{\prime \prime}$ each $0.144 \mathrm{~mm}$ to meet the $J$ band requirement of $0.125^{\prime \prime} /$ pixel sampling for background limited noise performance. This is a factor of 7 reduction in plate scale; because GMT is an F/8 telescope this therefore implies that the SuperFIRE cameras must operate at approximately F/1.0.

Such fast cameras are challenging, particularly in the NIR where (a) glass selection is more limited than at optical wavelengths, (b) elements must be air spaced to avoid CTE-induced stress, and (c) alignment must be held over a large swing from ambient $(295 \mathrm{~K})$ to operational (100K) temperature. These considerations led us to relax the pixel sampling requirement to $0.10^{\prime \prime}$ for $H$ and especially $K$, where thermal emission and the blended wings of hydroxyl lines exceed the inter-line continuum background. ${ }^{3}$ However the $y J$ band-which is critical for EoR science - has low $\mathrm{OH}$ contamination, and a very low continuum background, so the F/1.0 camera is a strict requirement.

We have additionally constrained our design study to use dioptric/refracting cameras in consideration of throughput. During GMT's early operations, instruments must deliver maximum functionality with a partial complement of primary mirror segments, one of which must be the center segment for proper phasing of the telescope. This makes catadioptric cameras unattractive, because their central obscuration for an H2RG sensor vignettes an entire 8.5 meter primary mirror segment. This can be mitigated by using a larger beam, but only with undesirable implications for the size, and by extension the cost and complexity of the instrument.

Figure 3 illustrates our early conceptual design for the F/1.0 J band camera, which is the fastest of the three units. It uses nine elements, primarily Ohara I-line glasses and $\mathrm{CaF}_{2}$, and all elements are airspaced with spherical surfaces. Because the most ambitious channel is at the blue end of SuperFIRE's bandpass (close to optical wavelengths), the selection of appropriate glasses is larger than in $K$, and does not incorporate glasses 


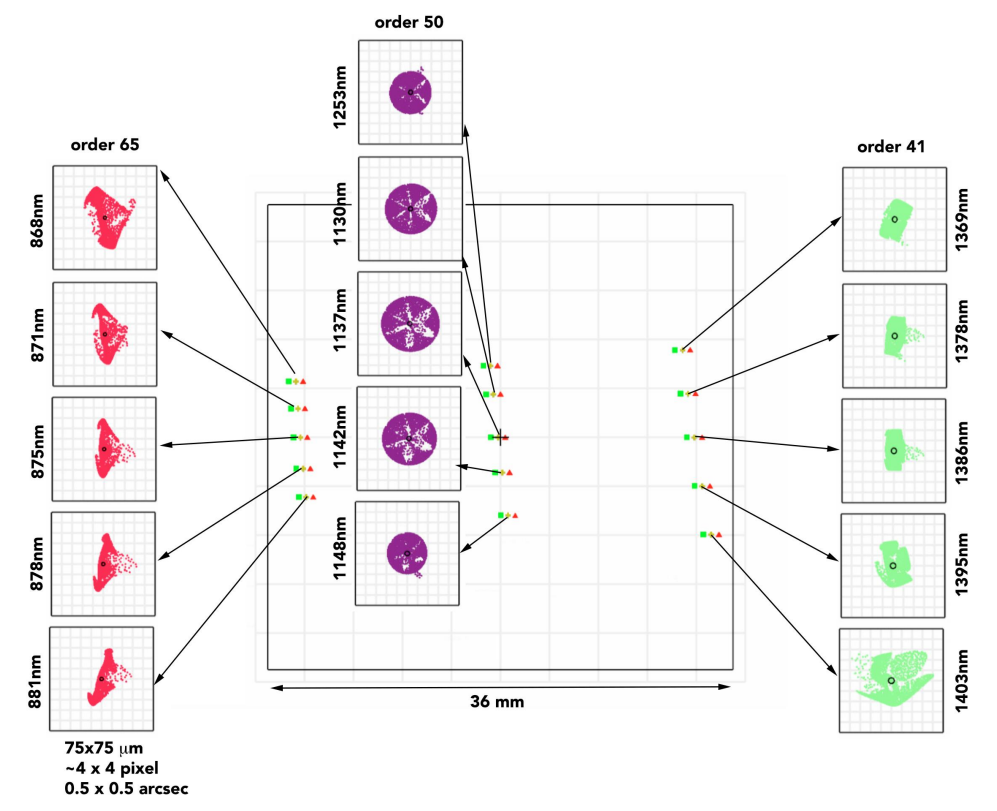

Figure 4. Footprint diagram for the $J$ band camera along with inset spot diagrams indicating performance along different portions of the echelle trace. Here, the inset boxes are $0.5^{\prime \prime}$ or 4 pixels per side. The $90 \%$ encircled energy diameter is $0.2^{\prime \prime}$ for all combinations except the reddest wavelength, or roughly $30 \%$ of the median seeing. Further optimization of the camera through use of aspheres should improve these preliminary results, which are intended only as a proof-of-concept that a solution exists for the most complex camera in the system.

such as S-FTM-16 that are often seen in IR cameras. In fact the design is derived from existing red optical cameras, with particular influence from the cameras for $\mathrm{G}_{-} \mathrm{Clef}^{2}$ and KCWI. ${ }^{30}$

Because the camera views a white pupil from a diffraction limited spectrograph, it may be optimized (and eventually tested) separately from the rest of the instrument given appropriate pairings of field angle and wavelength. We specified the lens in this context, but then evaluated its optical performance re-inserted into the spectrograph. The results are shown in Figure 4, which depicts spot diagrams along the central and two extreme orders of the $y J$ camera.

In contrast to Figure 2, the inset boxes are now $0.5^{\prime \prime}$ square, or approximately 4 pixels on a side. Typical seeing at the Magellan telescopes is $0.65-0.70^{\prime \prime}$ for reference. Clearly the aberrations are now dominated by the camera, but the $90 \%$ encircled energy is $\leq 0.2^{\prime \prime}$ for all angles except the extreme red at 1400nm (which is obscured in any case by atmospheric absorption).

This design is still in a preliminary phase, as very minimal effort has been made to optimize glass selection, and we have withheld the use of aspheric surfaces. We therefore view this as a proof-of-concept that an end-toend system can be built for the most difficult spectral channel, using COTS gratings and detectors, and optics with $\leq 120 \mathrm{~mm}$ diameter. We expect some improvement in spectral image quality once a full optimization is performed.

\section{MECHANICAL AND THERMAL DESIGN}

To verify compliance with mass and volume requirements imposed by GMT, and to estimate thermal loads, we developed a conceptual mechanical model for SuperFIRE. The spectrograph mounts on GMT's Instrument Platform, which sits below the telescope primary and is fed GMT's F/8 Gregorian beam via a diagonal tertiary. The entire Instrument Platform rotates about the optical axis of the telescope to track position angle; four or 

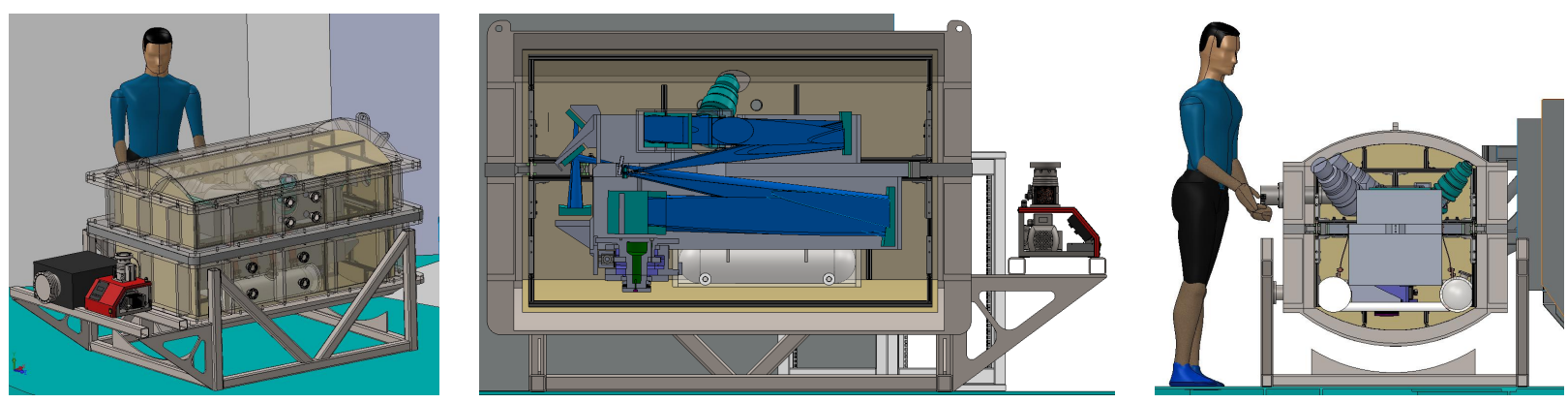

Figure 5. Mechanical model for SuperFIRE developed to estimate instrument footprint, volume envelope, and mass budget. Left panel illustrates the approximate size for scale, while the center panel (cross sectioned) illustrates the orientation and packaging of optical beams in the vacuum enclosure with radiation shield. Also visible in this panel is a cross-section of the turret spindle which configures the instrument between Echelle and Low-dispersion modes. The right panel shows a cutaway from the back, indicating the positions of the two LN2 reservoirs at the bottom of the cryostat. These chambers have capacity to deliver a hold time of 48 hours, but ample space is available in the dewar to accommodate larger reservoir volumes for additional margin. SuperFIRE's compact design (for a seeing-limited ELT instrument) easily meets volume envelope and mass requirements for GMT.

more instruments can be accommodated simultaneously and exchanged with minimal downtime. Guiding and wavefront sensing is managed by a separate optical package that is integral to the telescope. SuperFIRE therefore sees a variable gravity vector and must be designed with this dynamical environment in mind.

\subsection{Mechanical Envelope and Mass Budget}

Figure 5 shows three different views of the dewar volume, with a human figure for scale. The detailed attention to compactness of the optical design results in a cryostat that has a footprint of $1.8 \times 1.5$ meters, and height (including the integral handling fixture) of 1.2 meters. Including extra space on the handling fixture for pumping stations and calibration equipment not in vacuum, the total length grows to $\sim 2$ meters. This is only a factor of $\sim 2$ larger than FIRE on Magellan, and it is considerably smaller than many multi-object cryogenic spectrographs on 6-10 meter telescopes today. ${ }^{31,32}$ Since SuperFIRE is a single-object spectrograph, such a comparison does not imply that these instruments address identical science. It simply illustrates that fabrication, installation, and telemetry for dewars of this size are within current capabilities.

We estiamte an upper bound $220 \mathrm{~kg}$ for SuperFIRE's cold mass using a 6061-T6 Al model of the optical bench, optics (including three cameras), conceptual detector housing, and scaled up mechanical designs for the grating/mirror exchange mechanism and slit selector employed in FIRE. This estimate includes a very conservative $5 \mathrm{~cm}$ thickness for the optical bench, which has not been lightweighted (which reduced FIRE's optical bench mass by a factor of 2 ).

The optical bench concept is shown in Figure 6. The white pupil design is naturally symmetric about a midplane between M1 and M3, which defines the location of the bench. Individual optical subassemblies bolt to either side of this unit, which is suspended in the vacuum enclosure by G10 flexures aligned to intersect the center of mass. An outer ring which supports the bench indexes to the instrument handling fixture, eliminating optical displacements associated with deformations of the dewar walls when the instrument is under vacuum.

A binary turret mechanism identical to FIRE's is used to switch betwen echelle and low-dispersion spectroscopic modes. This system uses a worm gear driven by a small flexure stage which displaces and triggers a mechanical limit switch when in position. In those positions, which are defined by the mechanical hard stops, the turret is held in position by the axial preload on the worm shaft provided by the flexure stage.

The total mass of this conceptual model is $1350 \mathrm{~kg}$, which easily satisfies GMT's requirement of $\leq 7000 \mathrm{~kg}$ for Instrument Platform installations. Figure 7 shows SuperFIRE mounted on the rotating disk of the Instrument Platform. The allowed volume envelope for instruments is indicated for the other three quadrants (one rendered transparent), illustrating that SuperFIRE fits very comfortably within this space with substantial margin and additional room for equipment racks. Figure 8 presents renderings of the instrument in the context of the 

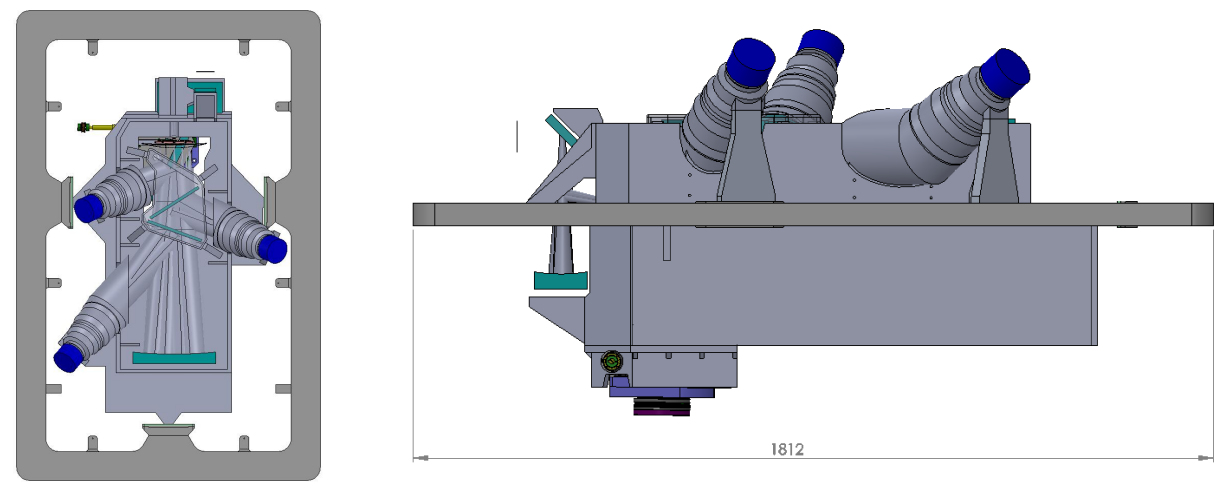

Figure 6. Cold mass and support structure for SuperFIRE, with critical outside dimensions that define the outer instrument envelope. The outer support ring features O-ring seals on both sides, allowing the instrument to be articulated during image plane and pupil plane alignment from the exterior. It also allows servicing access to both sides of the instrument while mounted on the handling fixture.
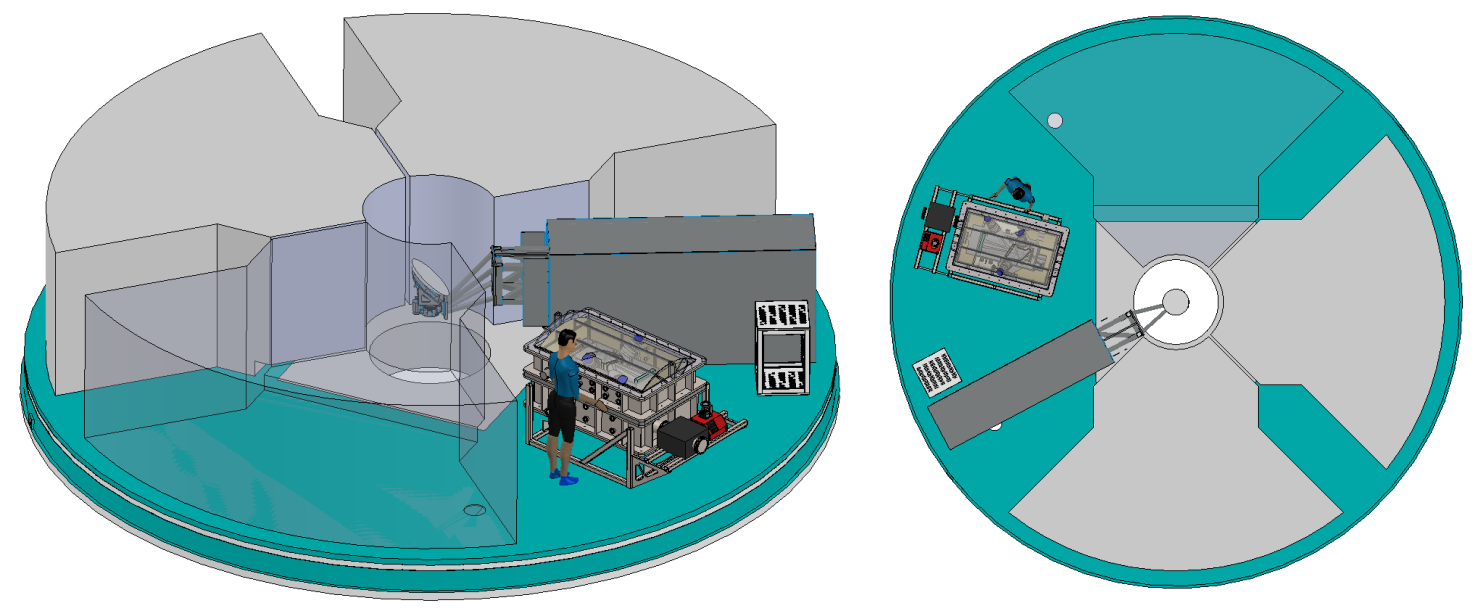

Figure 7. SuperFIRE shown on the rotating GMT Instrument Platform for scale. The tertiary shown in the figure is a preliminary design from GMT; SuperFIRE would instead use a dichroic tertiary that transmits optical light through the platform aperture to Direct Gregorian instruments below. For reference, the transparent solid indicates GMT's allocated instrument envelope at these stations as defined in the telescope ICD.

full GMT, showing the instrument location underneath the primary mirror array. Access for installation and servicing is available via a lift that meets the space between GMT's elevation bearings.

\subsection{Thermal Loads and Design Strategy}

We used the mechanical model described above to estimate thermal loads on the cryostat and components. We calculate the radiative load assuming an operating temperature of $T=100 \mathrm{~K}$ and a single-layer passive radiation shield of aluminized kapton, suspended between the dewar exterior and cold mass. We include conductive losses through the support structure and an estimate for mechanical and electrical feedthroguhs. From these sources we estimate a total analytic heat load of $20 \mathrm{~W}$, with the majority derived from radiation. For margin and from comparison with other instruments we allocate a budget of $80 \mathrm{~W}$ to the cooling system.

Because GMT will be commissioning an advanced adaptive optics system with multiple deformable secondary mirrors during SuperFIRE's scientific operations, the observatory has restricted use of mechanical cryocoolers in early instrument packages. Our cooling architecture therefore employs LN2 reservoirs which plumb into an observatory feed. The dewar easily accommodates two 40L chambers below the optical bench, on either side of the grating turret, providing 1.5-2 day hold time. LN2 fills will be performed during the day for thermal stability 


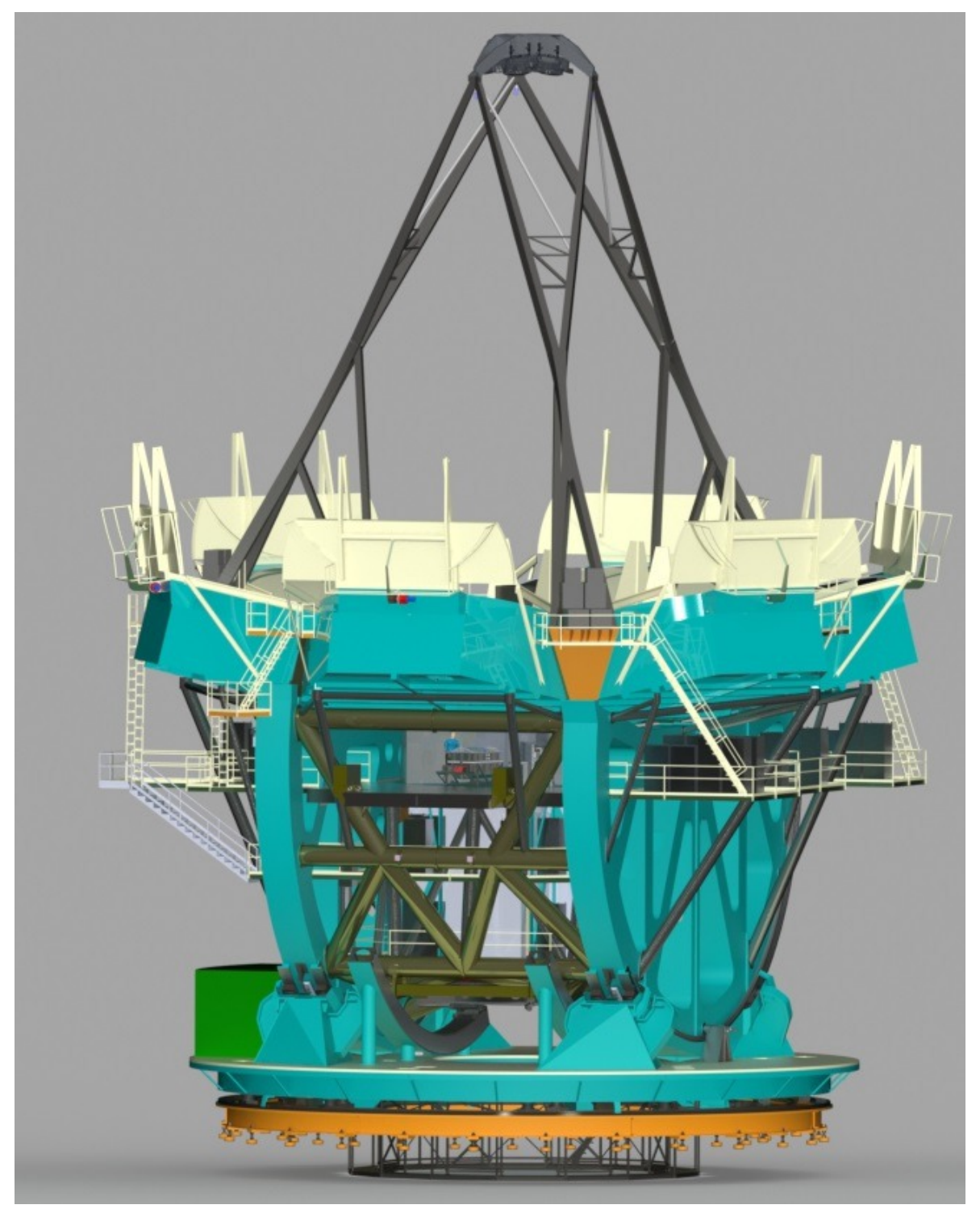

Figure 8. SuperFIRE as mounted on GMT's Instrument Platform. The platform is located under the primary mirror, where SuperFIRE can be seen again with a human figure for scale. Access for installation is available via an elevator platform between the telescope's elevation bearings.

and to minimize vibrations during nighttime operations. No other special infrastruture is needed to operate the instrument.

\subsection{Low Dispersion Mode}

The Magellan/FIRE design includes a Low-Dispersion mode with $R \sim 400$ and a $50^{\prime \prime}$ longslit which has been extremely popular among observers. It is extremely efficient for spectral classification, and for faint objects requiring high sensitivity but low requirements on velocity resolution: e.g. low-mass stars, and transient events such as supernovae and gamma-ray bursts.

FIRE is changed into this configuration by replacing the echelle grating with a flat mirror and re-orienting the slit by $90^{\circ}$, repurposing the prisms normally used for cross-dispersion as the primary disperser. Although the 


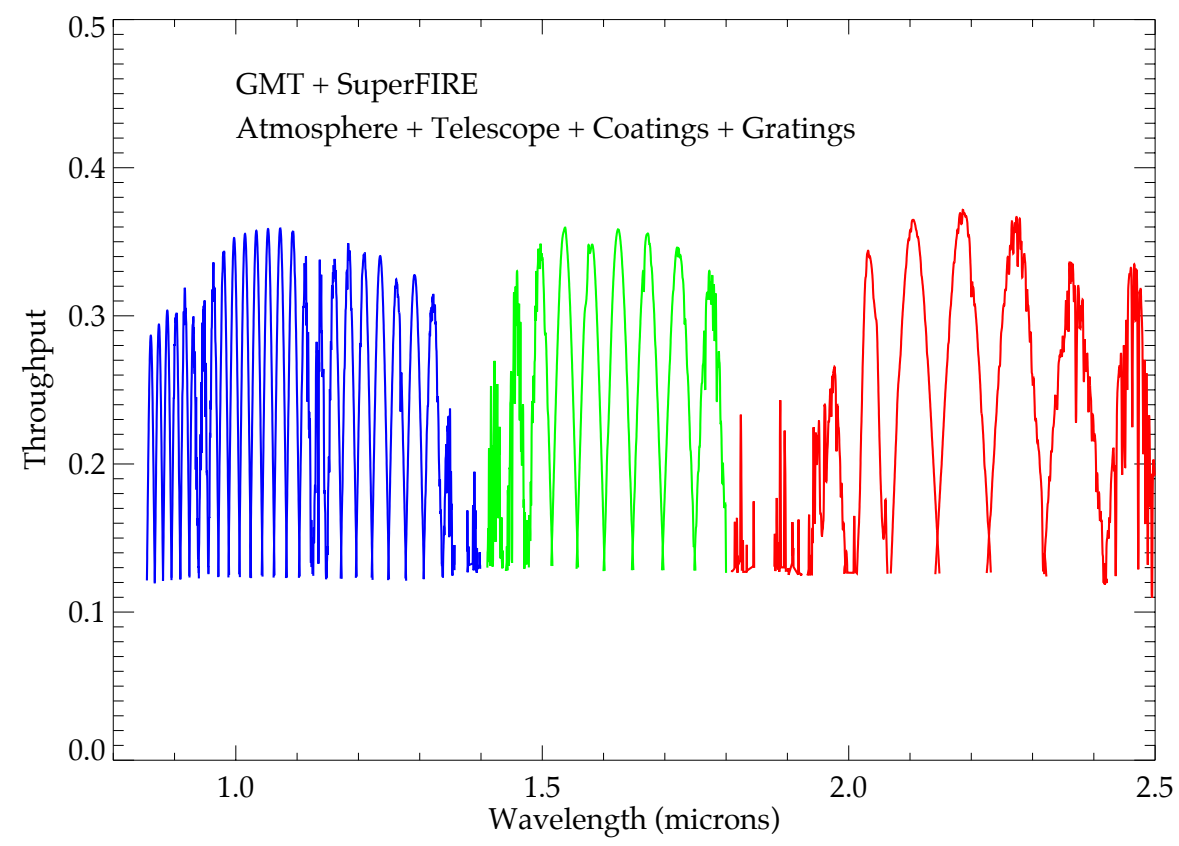

Figure 9. Projected throughput of SuperFIRE, including losses from the Earth's atmosphere, telescope, and instrument. The instrument contribution consists of optical surface losses, blaze functions of the echelle grating and VPH cross dispersers (modeled analytically using the Kogelnik approximation), and the H2RG sensor's quantum efficiency. No slit losses are included. This throughput is similar to Magellan/FIRE at similar plate scale, so GMT's aperture should deliver large improvements in system sensitivity. This is particularly true in $y J$ and portions of $H$, where FIRE is read-noise limited on Magellan.

spectral resolution is substantially reduced, the sensitivity is extremely high and data reduction is particularly simple.

Given the importance of these science applications for GMT, and the possibility of fast and broadband transient observations, we wish to offer a similar capability with SuperFIRE. Because the bandpass is similar to FIRE but spread over three H2RG sensors rather than one, the spectral resolution delivered naturally by the cross dispersers is roughly twice as high, or $R \sim 800$. This somewhat higher dispersion is advantageous because Magellan/FIRE is often limited in duty cycle by saturation of the sky in $K$ and associated readout. The $12 \times$ larger collecting area of GMT relative to Magellan (which would saturate in seconds at $R=400$ ) is partially offset by SuperFIRE's higher resolution and further mitigated by the ablity to reset the $K$ sensor more frequently than $y J$ and $H$ since (unlike FIRE) the detectors are operated in parallel.

A disadvantage of SuperFIRE's low-dispersion mode is its relatively shorter slit, at just $15-20^{\prime \prime}$. Although the Offner relay accommodates a larger field of view, the compactness of the white pupil design is specific to the dispersion direction of the echelle grating. Although angles diffracting from the echelle appear qualitatively similar to geometric field angles from a long slit at M2 (the Mangin mirror), a full analysis reveals that rays from the longslit edges are displaced at the white pupil, leading to vignetting of the slit edges by the camera aperture. While this could theoretically be mitigated by increasing the camera size, in SuperFIRE the $J$ camera is already operating at F/1.0 so this solution is impractical.

For our nominal $120 \mathrm{~mm}$ camera aperture, SuperFIRE accomodates a $20^{\prime \prime}$ slit length with a maximum of $10 \%$ vignetting at the extreme ends of the slit. Longer slits are desirable but are not practial without extremely fast cameras that are not consistent with the low-cost, simple philosopy of SuperFIRE's design. 


\section{PROJECTED THROUGHPUT}

In Figure 9 we model SuperFIRE's throughput using assumptions about optical coatings and grating selection. The blue, green, and red curves correspond to SuperFIRE's three camera channels, which are separated by atmospheric absorption bands as indicated. This model is representative of sky-to-detector efficiency, in that it includes attenuation from the Earth's atmosphere, three reflections from GMT ( $87 \%$ each), as-measured performance of optical coatings and H2RG sensors, and analytic models of grating efficiency as described below.

Our atmospheric absorption model is taken from measurements made at Las Campanas using FIRE, scaled to an effective airmass of 1.2. Dielectric coating data for lenses and windows are taken from measured, as-delivered curves for either identical or similar-index glasses used in FIRE, from Evaporated Coatings Inc. Of these glasses, the main contributor to throughput loss is in the CaF2 camera lenses, which were coated for the full $0.8-2.5 \mu \mathrm{m}$ bandpass in FIRE but can be narrowed in wavelength for SuperFIRE's three arms to imrove performance. All reflecting surfaces were assumed to be coated with protected gold, with as-built reflectivity measurements made from FIRE's optics. We use quantum efficiency measurements from the identical H2RG sensor in use on FIRE.

To estimate the efficiency of the echelle grating, we generate an analytic model of the blaze function based on the ruling density and blaze angle to capture variation in throughput along each order. The throughput at blaze peak is then normalized using efficiency data tabulated by the Richardson Grating Lab at the orders of interest. For our preferred master, which can accommodate the full size of SuperFIRE's ruling, this peak efficiency is near 80\%. As usual for echelles, the drop in efficiency near the edge of the FSR for any one order may be compensated by combining flux at the same wavelength in adjacent orders to flatten the throghput curve, which we have not done for this figure. This requires that the sensor area extend substantially beyond the edge of the free spectral range to capture photons off the blaze peak, but Figure 1 illustrates that SuperFIRE's echelle footprint meets this criterion in all regions except the reddest orders in $K$.

To estimate the VPH blaze efficiency, for this trade study, we use the analytic Kogelnik ${ }^{33}$ approximations for the VPH ruling densities required, and typical assumptions about refractive index mean, varation, and gel thickness. This first analysis confirms that VPH gratings can indeed maintain a high cross-dispersion efficiency if split into the three channels, and also guides us in selection of parameter space for a mode detailed study. The next step is to confirm this performance via rigorous coupled wave analysis.

The only significant effect not included in this throguhput calculation is slit loss, which is minimized through use of a $0.7^{\prime \prime}$ fiduacial slit. For reference, this would result in an additional $\sim 35 \%$ penalty if the seeing FWHM is matched to the slit width.

The overall efficiency of the atmosphere, telescope, and instrument is $\sim 35 \%$ at blaze peak. This value is similar to FIRE's as-built throughput, which may at first be surprising because FIRE's F/3.5 camera has only four elements to SuperFIRE's nine. However FIRE's cross-dispersion requires six prism passes at high incidence angle relative SuperFIRE's single VPH. This makes the total number of optical elements approximately the same, resulting in a similar total efficiency.

With its similar projected throughput, pixel scale, and spectral resolution, SuperFIRE should therefore be well positioned to capitalize on GMT's twelve-fold increase in collecting area relative to Magellan. More specifically, because FIRE is read noise limited, even in the seeing limit SuperFIRE should achieve up to a twelve-fold gain in signal-to-noise per integration. This is much more favorable than the factor of $\sim 3.5$ gain in SNR for optical instruments that are already sky-noise limited on current-generation telescopes, indicating in part why NIR spectroscopy — even in the seeing limit - should see large performance gains in the era of ELTs.

\section{SUMMARY}

SuperFIRE is a concept for a single-object NIR spectrometer developed in response to the challenge of buiding compelling instruments at the ELT scale, in a tightly constrained budgetary environment. It further addresses the question of how GMT can maximize its impact on core science areas during a commissioning period, when the primary mirror is largely deployed but the Adaptive Optics system is not yet operational.

NIR spectrographs achieve particularly large gains from the increased collecting area of GMT, because spectrometers on current-generation telescopes operate near or below the read noise limit. While all ELTs will 
eventually be able to exploit advanced AO systems to achieve even greater performance gains in the NIR, the inclusion of a modest seeing-limited spectrometer fills an important gap in GMT's early science capabilities.

The science requirements and design parameters for SuperFIRE were heavily informed by our prior work with FIRE on Magellan. However the detailed design differs significantly, owing to the fabrication challenges for large cross-dispersion prisms, and the requirement of much faster F/1.0 spectrograph cameras for GMT realtive to Magellan.

These challenges are solved for SuperFIRE by moving to an asymmetric white pupil optical layout and higher blaze angle, which together limit the beam to $132 \mathrm{~mm}$ at the echelle and $120 \mathrm{~mm}$ at the camera's extrance pupil. The white pupil layout delivers diffraction limited wavefronts to the three cameras. We present a preliminary design for the most challenging of the three cameras, which operates in $J$ and has the fastest focal ratio. This 9 -element, all spherical camera produces images with $90 \%$ encircled energy in a $0.2^{\prime \prime}$ spot. Our near term efforts are focused on further improvements of this design through use of aspheric elements and further optimization of glass selection.

This layout leads to a modestly sized cryostat for an ELT instrument, that easily meets size and weight requrements for GMT. This fact, plus the extensive use of COTS design elements with astronomy heritage (e.g. H2RG sensors, catalog echelles, small optics) helps keep cost and schedule risk to a minimum. The predicted instrument efficiency is $\sim 35 \%$ at blaze peak, similar to FIRE.

Because SuperFIRE is fed by a tertiary optic and mounts on GMT's rotating Instrument Platform, one can in principle operate SuperFIRE with GMACS (GMT's planned optical low-resolution spectrometer) to achieve sumultaneous wavelength coverage from the ultraviolet throguh the NIR, similar to X-Shooter on the VLT. This is achieved by deploying a dichroic at the tertiary location so that optical light transmits to GMACS' direct Gregorian focal station below the Instrument Platform.

Installation of a fast, simple NIR spectrometer would position GMT to immediately pursue several key programs outlined in its Science Book that are not addressed with the current first-light instrument suite. These programs, which include observations of quasars and galaxies in the early universe/epoch of reionization, transient astrophysical sources, moderate redshift galaxies and circumgalactic gas, and star- and planet-formation sites that are heavily dust-obscured, have been highlighted in surveys of high impact early science for GMT produced by the Science Advisory Council.

It would also compliment the capabilities to NIRSPEC on JWST, because JWST's smaller aperture (equivalent to Magellan) will also put NIRSPEC in the read-noise limited regime except at extremely low spectral resolution. This suggests a role for GMT to study detailed the detailed astrophysics of faint sources at higher spectral resolutions, for objects discovered in low-resolution surveys with JWST — particularly in the $y$ and $J$ bands where terrestrial sky backgrounds are low.

\section{ACKNOWLEDGMENTS}

This work was supported by the MIT Department of Physics through the Francis L. Friedman Chair, and also by the MIT Kavli Institute for Astrophsyics and Space Research. We gratefully acknowledge support and helpful conversations with Stephen Shectman, Hsiao-Wen Chen, Rebecca Bernstein, George Jacoby, Daniel Fabricant, and other members of the GMT Science Advisory Committee.

\section{REFERENCES}

[1] DePoy, D. L., Allen, R., Barkhouser, R., Boster, E., Carona, D., Harding, A., Hammond, R., Marshall, J. L., Orndorff, J., Papovich, C., Prochaska, K., Prochaska, T., Rheault, J. P., Smee, S., Shectman, S., and Villanueva, S., "GMACS: a wide field, multi-object, moderate-resolution, optical spectrograph for the Giant Magellan Telescope," in [Ground-based and Airborne Instrumentation for Astronomy IV], Proc. of the SPIE 8446, 84461N (Sept. 2012). 
[2] Szentgyorgyi, A., Barnes, S., Bean, J., Bigelow, B., Bouchez, A., Chun, M.-Y., Crane, J. D., Epps, H., Evans, I., Evans, J., Frebel, A., Furesz, G., Glenday, A., Guzman, D., Hare, T., Jang, B.-H., Jang, J.-G., Jeong, U., Jordan, A., Kim, K.-M., Kim, J., Li, C.-H., Lopez-Morales, M., McCracken, K., McLeod, B., Mueller, M., Nah, J., Norton, T., Oh, H., Oh, J. S., Ordway, M., Park, B.-G., Park, C., Park, S.-J., Phillips, D., Plummer, D., Podgorski, W., Rodler, F., Seifahrt, A., Tak, K.-M., Uomoto, A., Van Dam, M. A., Walsworth, R., Yu, Y. S., and Yuk, I.-S., "A preliminary design for the GMT-Consortium Large Earth Finder (G-CLEF)," in [Ground-based and Airborne Instrumentation for Astronomy V], Proc. of the SPIE 9147, 914726 (Aug. 2014).

[3] Sullivan, P. W. and Simcoe, R. A., "A Calibrated Measurement of the Near-IR Continuum Sky Brightness Using Magellan/FIRE," Publications of the Astronomical Society of the PAcific 124, 1336-1346 (Dec. 2012).

[4] Jaffe, D. T., Mar, D. J., Warren, D., and Segura, P. R., "GMTNIRS: the high resolution near-IR spectrograph for the Giant Magellan Telescope," in [Society of Photo-Optical Instrumentation Engineers (SPIE) Conference Series], Proc. of the SPIE 6269, 62694I (June 2006).

[5] McGregor, P. J., Bloxham, G. J., Boz, R., Davies, J., Doolan, M., Ellis, M., Hart, J., Jones, D. J., Luvaul, L., Nielsen, J., Parcell, S., Sharp, R., Stevanovic, D., and Young, P. J., "GMT integral-field spectrograph (GMTIFS) conceptual design," in [Ground-based and Airborne Instrumentation for Astronomy IV], Proc. of the SPIE 8446, 84461I (Sept. 2012).

[6] Simcoe, R. A., Burgasser, A. J., Schechter, P. L., Fishner, J., Bernstein, R. A., Bigelow, B. C., Pipher, J. L., Forrest, W., McMurtry, C., Smith, M. J., and Bochanski, J. J., "FIRE: A Facility Class NearInfrared Echelle Spectrometer for the Magellan Telescopes," Publications of the Astronomical Society of the PAcific 125, 270-286 (Mar. 2013).

[7] McCarthy, P. J. and the GMT Science Advisory Council, [The Giant Magellan Telescope: Scientific Promise and Opportunities] (2012).

[8] Planck Collaboration, Adam, R., Aghanim, N., Ashdown, M., Aumont, J., Baccigalupi, C., Ballardini, M., Banday, A. J., Barreiro, R. B., Bartolo, N., Basak, S., Battye, R., Benabed, K., Bernard, J.-P., Bersanelli, M., Bielewicz, P., Bock, J. J., Bonaldi, A., Bonavera, L., Bond, J. R., Borrill, J., Bouchet, F. R., Bucher, M., Burigana, C., Calabrese, E., Cardoso, J.-F., Carron, J., Chiang, H. C., Colombo, L. P. L., Combet, C., Comis, B., Coulais, A., Crill, B. P., Curto, A., Cuttaia, F., Davis, R. J., de Bernardis, P., de Rosa, A., de Zotti, G., Delabrouille, J., Di Valentino, E., Dickinson, C., Diego, J. M., Doré, O., Douspis, M., Ducout, A., Dupac, X., Elsner, F., Enßlin, T. A., Eriksen, H. K., Falgarone, E., Fantaye, Y., Finelli, F., Forastieri, F., Frailis, M., Fraisse, A. A., Franceschi, E., Frolov, A., Galeotta, S., Galli, S., Ganga, K., Génova-Santos, R. T., Gerbino, M., Ghosh, T., González-Nuevo, J., Górski, K. M., Gruppuso, A., Gudmundsson, J. E., Hansen, F. K., Helou, G., Henrot-Versillé, S., Herranz, D., Hivon, E., Huang, Z., Ili, S., Jaffe, A. H., Jones, W. C., Keihänen, E., Keskitalo, R., Kisner, T. S., Knox, L., Krachmalnicoff, N., Kunz, M., Kurki-Suonio, H., Lagache, G., Lähteenmäki, A., Lamarre, J.-M., Langer, M., Lasenby, A., Lattanzi, M., Lawrence, C. R., Le Jeune, M., Levrier, F., Lewis, A., Liguori, M., Lilje, P. B., López-Caniego, M., Ma, Y.-Z., Macías-Pérez, J. F., Maggio, G., Mangilli, A., Maris, M., Martin, P. G., Martínez-González, E., Matarrese, S., Mauri, N., McEwen, J. D., Meinhold, P. R., Melchiorri, A., Mennella, A., Migliaccio, M., Miville-Deschênes, M.-A., Molinari, D., Moneti, A., Montier, L., Morgante, G., Moss, A., Naselsky, P., Natoli, P., Oxborrow, C. A., Pagano, L., Paoletti, D., Partridge, B., Patanchon, G., Patrizii, L., Perdereau, O., Perotto, L., Pettorino, V., Piacentini, F., Plaszczynski, S., Polastri, L., Polenta, G., Puget, J.-L., Rachen, J. P., Racine, B., Reinecke, M., Remazeilles, M., Renzi, A., Rocha, G., Rossetti, M., Roudier, G., Rubiño-Martín, J. A., Ruiz-Granados, B., Salvati, L., Sandri, M., Savelainen, M., Scott, D., Sirri, G., Sunyaev, R., Suur-Uski, A.-S., Tauber, J. A., Tenti, M., Toffolatti, L., Tomasi, M., Tristram, M., Trombetti, T., Valiviita, J., Van Tent, F., Vielva, P., Villa, F., Vittorio, N., Wandelt, B. D., Wehus, I. K., White, M., Zacchei, A., and Zonca, A., "Planck intermediate results. XLVII. Planck constraints on reionization history," ArXiv e-prints (May 2016).

[9] Parsons, A. R., Liu, A., Aguirre, J. E., Ali, Z. S., Bradley, R. F., Carilli, C. L., DeBoer, D. R., Dexter, M. R., Gugliucci, N. E., Jacobs, D. C., Klima, P., MacMahon, D. H. E., Manley, J. R., Moore, D. F., Pober, J. C., Stefan, I. I., and Walbrugh, W. P., "New Limits on $21 \mathrm{~cm}$ Epoch of Reionization from PAPER-32 Consistent with an X-Ray Heated Intergalactic Medium at $\mathrm{z}=7.7$," The Astrophysical Journal 788, 106 (June 2014).

[10] Greig, B. and Mesinger, A., "The Global History of Reionisation," ArXiv e-prints (May 2016). 
[11] Fan, X., Narayanan, V. K., Lupton, R. H., Strauss, M. A., Knapp, G. R., Becker, R. H., White, R. L., Pentericci, L., Leggett, S. K., Haiman, Z., Gunn, J. E., Ivezić, Ž., Schneider, D. P., Anderson, S. F., Brinkmann, J., Bahcall, N. A., Connolly, A. J., Csabai, I., Doi, M., Fukugita, M., Geballe, T., Grebel, E. K., Harbeck, D., Hennessy, G., Lamb, D. Q., Miknaitis, G., Munn, J. A., Nichol, R., Okamura, S., Pier, J. R., Prada, F., Richards, G. T., Szalay, A., and York, D. G., "A Survey of $\mathrm{z} \geq 5.8$ Quasars in the Sloan Digital Sky Survey. I. Discovery of Three New Quasars and the Spatial Density of Luminous Quasars at z 6," The Astronomical Journal 122, 2833-2849 (Dec. 2001).

[12] Stark, D. P., Richard, J., Charlot, S., Clément, B., Ellis, R., Siana, B., Robertson, B., Schenker, M., Gutkin, J., and Wofford, A., "Spectroscopic detections of C III] $\lambda 1909 \AA$ at $z \sim 6-7$ : a new probe of early star-forming galaxies and cosmic reionization," Monthly Notices of the Royal Astronomical Society 450, 1846-1855 (June 2015).

[13] Finkelstein, S. L., Papovich, C., Dickinson, M., Song, M., Tilvi, V., Koekemoer, A. M., Finkelstein, K. D., Mobasher, B., Ferguson, H. C., Giavalisco, M., Reddy, N., Ashby, M. L. N., Dekel, A., Fazio, G. G., Fontana, A., Grogin, N. A., Huang, J.-S., Kocevski, D., Rafelski, M., Weiner, B. J., and Willner, S. P., "A galaxy rapidly forming stars 700 million years after the Big Bang at redshift 7.51," Nature 502, 524-527 (Oct. 2013).

[14] Bouwens, R. J., Bradley, L., Zitrin, A., Coe, D., Franx, M., Zheng, W., Smit, R., Host, O., Postman, M., Moustakas, L., Labbé, I., Carrasco, M., Molino, A., Donahue, M., Kelson, D. D., Meneghetti, M., Benítez, N., Lemze, D., Umetsu, K., Broadhurst, T., Moustakas, J., Rosati, P., Jouvel, S., Bartelmann, M., Ford, H., Graves, G., Grillo, C., Infante, L., Jimenez-Teja, Y., Lahav, O., Maoz, D., Medezinski, E., Melchior, P., Merten, J., Nonino, M., Ogaz, S., and Seitz, S., "A Census of Star-forming Galaxies in the Z 9-10 Universe based on HST+Spitzer Observations over 19 Clash Clusters: Three Candidate Z 9-10 Galaxies and Improved Constraints on the Star Formation Rate Density at Z 9.2," The Astrophysical Journal 795, 126 (Nov. 2014).

[15] Fan, X., Strauss, M. A., Becker, R. H., White, R. L., Gunn, J. E., Knapp, G. R., Richards, G. T., Schneider, D. P., Brinkmann, J., and Fukugita, M., "Constraining the Evolution of the Ionizing Background and the Epoch of Reionization with z $\sim 6$ Quasars. II. A Sample of 19 Quasars," The Astronomical Journal 132, 117-136 (July 2006).

[16] Becker, R. H., Fan, X., White, R. L., Strauss, M. A., Narayanan, V. K., Lupton, R. H., Gunn, J. E., Annis, J., Bahcall, N. A., Brinkmann, J., Connolly, A. J., Csabai, I., Czarapata, P. C., Doi, M., Heckman, T. M., Hennessy, G. S., Ivezić, Ž., Knapp, G. R., Lamb, D. Q., McKay, T. A., Munn, J. A., Nash, T., Nichol, R., Pier, J. R., Richards, G. T., Schneider, D. P., Stoughton, C., Szalay, A. S., Thakar, A. R., and York, D. G., "Evidence for Reionization at $\mathrm{z}^{\sim} 6$ : Detection of a Gunn-Peterson Trough in a $\mathrm{z}=6.28$ Quasar," The Astronomical Journal 122, 2850-2857 (Dec. 2001).

[17] Mortlock, D. J., Warren, S. J., Venemans, B. P., Patel, M., Hewett, P. C., McMahon, R. G., Simpson, C., Theuns, T., Gonzáles-Solares, E. A., Adamson, A., Dye, S., Hambly, N. C., Hirst, P., Irwin, M. J., Kuiper, E., Lawrence, A., and Röttgering, H. J. A., "A luminous quasar at a redshift of $\mathrm{z}=7.085$," Nature 474, 616-619 (June 2011).

[18] Simcoe, R. A., Sullivan, P. W., Cooksey, K. L., Kao, M. M., Matejek, M. S., and Burgasser, A. J., "Extremely metal-poor gas at a redshift of 7," Nature 492, 79-82 (Dec. 2012).

[19] Becker, G. D., Bolton, J. S., and Lidz, A., "Reionisation and High-Redshift Galaxies: The View from Quasar Absorption Lines," Publications of the Astronomical Society of Australia 32, e045 (Dec. 2015).

[20] Kasliwal, M., Cao, Y., Masci, F., Helou, G., Williams, R., Bally, J., Bond, H., Whitelock, P., Cody, A. M., Gehrz, R., Jencson, J., Tinyanont, S., Smith, N., Surace, J., Armus, L., Cantiello, M., Langer, N., Levesque, E., Mohamed, S., Ofek, E., Parthasarathy, M., van Dyk, S., Boyer, M., Phillips, M., Hsiao, E., Morrell, N., Perley, D., Gonzalez, C., and Contreras, C., "SPIRITS: SPitzer InfraRed Intensive Transients Survey." Spitzer Proposal (Dec. 2014).

[21] Smith, N., Andrews, J. E., Van Dyk, S. D., Mauerhan, J. C., Kasliwal, M. M., Bond, H. E., Filippenko, A. V., Clubb, K. I., Graham, M. L., Perley, D. A., Jencson, J., Bally, J., Ubeda, L., and Sabbi, E., "Massive star mergers and the recent transient in NGC 4490: a more massive cousin of V838 Mon and V1309 Sco," Monthly Notices of the Royal Astronomical Society 458, 950-962 (May 2016). 
[22] Abbott, B. P., Abbott, R., Abbott, T. D., Abernathy, M. R., Acernese, F., Ackley, K., Adams, C., Adams, T., Addesso, P., Adhikari, R. X., and et al., "Observation of Gravitational Waves from a Binary Black Hole Merger," Physical Review Letters 116, 061102 (Feb. 2016).

[23] Metzger, B. D. and Berger, E., "What is the Most Promising Electromagnetic Counterpart of a Neutron Star Binary Merger?," The Astrophysical Journal 746, 48 (Feb. 2012).

[24] Kasen, D., Badnell, N. R., and Barnes, J., "Opacities and Spectra of the r-process Ejecta from Neutron Star Mergers," The Astrophysical Journal 774, 25 (Sept. 2013).

[25] van Dokkum, P. G., Nelson, E. J., Franx, M., Oesch, P., Momcheva, I., Brammer, G., Förster Schreiber, N. M., Skelton, R. E., Whitaker, K. E., van der Wel, A., Bezanson, R., Fumagalli, M., Illingworth, G. D., Kriek, M., Leja, J., and Wuyts, S., "Forming Compact Massive Galaxies," The Astrophysical Journal 813, 23 (Nov. 2015).

[26] Barro, G., Faber, S. M., Pérez-González, P. G., Pacifici, C., Trump, J. R., Koo, D. C., Wuyts, S., Guo, Y., Bell, E., Dekel, A., Porter, L., Primack, J., Ferguson, H., Ashby, M. L. N., Caputi, K., Ceverino, D., Croton, D., Fazio, G. G., Giavalisco, M., Hsu, L., Kocevski, D., Koekemoer, A., Kurczynski, P., Kollipara, P., Lee, J., McIntosh, D. H., McGrath, E., Moody, C., Somerville, R., Papovich, C., Salvato, M., Santini, P., Tal, T., van der Wel, A., Williams, C. C., Willner, S. P., and Zolotov, A., "CANDELS+3D-HST: Compact SFGs at z 2-3, the Progenitors of the First Quiescent Galaxies," The Astrophysical Journal 791, 52 (Aug. 2014).

[27] Shapley, A. E., Reddy, N. A., Kriek, M., Freeman, W. R., Sanders, R. L., Siana, B., Coil, A. L., Mobasher, B., Shivaei, I., Price, S. H., and de Groot, L., "The MOSDEF Survey: Excitation Properties of $z \sim 2.3$ Star-forming Galaxies," The Astrophysical Journal 801, 88 (Mar. 2015).

[28] Vernet, J., Dekker, H., D’Odorico, S., Kaper, L., Kjaergaard, P., Hammer, F., Randich, S., Zerbi, F., Groot, P. J., Hjorth, J., Guinouard, I., Navarro, R., Adolfse, T., Albers, P. W., Amans, J.-P., Andersen, J. J., Andersen, M. I., Binetruy, P., Bristow, P., Castillo, R., Chemla, F., Christensen, L., Conconi, P., Conzelmann, R., Dam, J., de Caprio, V., de Ugarte Postigo, A., Delabre, B., di Marcantonio, P., Downing, M., Elswijk, E., Finger, G., Fischer, G., Flores, H., François, P., Goldoni, P., Guglielmi, L., Haigron, R., Hanenburg, H., Hendriks, I., Horrobin, M., Horville, D., Jessen, N. C., Kerber, F., Kern, L., Kiekebusch, M., Kleszcz, P., Klougart, J., Kragt, J., Larsen, H. H., Lizon, J.-L., Lucuix, C., Mainieri, V., Manuputy, R., Martayan, C., Mason, E., Mazzoleni, R., Michaelsen, N., Modigliani, A., Moehler, S., Møller, P., Norup Sørensen, A., Nørregaard, P., Péroux, C., Patat, F., Pena, E., Pragt, J., Reinero, C., Rigal, F., Riva, M., Roelfsema, R., Royer, F., Sacco, G., Santin, P., Schoenmaker, T., Spano, P., Sweers, E., Ter Horst, R., Tintori, M., Tromp, N., van Dael, P., van der Vliet, H., Venema, L., Vidali, M., Vinther, J., Vola, P., Winters, R., Wistisen, D., Wulterkens, G., and Zacchei, A., "X-shooter, the new wide band intermediate resolution spectrograph at the ESO Very Large Telescope," Astronomy and Astrophysics 536, A105 (Dec. 2011).

[29] Tull, R. G., MacQueen, P. J., Sneden, C., and Lambert, D. L., "The high-resolution cross-dispersed echelle white-pupil spectrometer of the McDonald Observatory 2.7-m telescope," Publications of the Astronomical Society of the Pacific 107, 251-264 (Mar. 1995).

[30] Morrissey, P., Matuszewski, M., Martin, C., Moore, A., Adkins, S., Epps, H., Bartos, R., Cabak, J., Cowley, D., Davis, J., Delacroix, A., Fucik, J., Hilliard, D., James, E., Kaye, S., Lingner, N., Neill, J. D., Pistor, C., Phillips, D., Rockosi, C., and Weber, B., "The Keck Cosmic Web Imager: a capable new integral field spectrograph for the W. M. Keck Observatory," in [Ground-based and Airborne Instrumentation for Astronomy IV], Proceedings of the SPIE 8446, 844613 (Sept. 2012).

[31] McLean, I. S., Steidel, C. C., Epps, H. W., Konidaris, N., Matthews, K. Y., Adkins, S., Aliado, T., Brims, G., Canfield, J. M., Cromer, J. L., Fucik, J., Kulas, K., Mace, G., Magnone, K., Rodriguez, H., Rudie, G., Trainor, R., Wang, E., Weber, B., and Weiss, J., "MOSFIRE, the multi-object spectrometer for infra-red exploration at the Keck Observatory," in [Ground-based and Airborne Instrumentation for Astronomy IV], Proceedings of the SPIE 8446, 84460J (Sept. 2012).

[32] Eikenberry, S., Elston, R., Raines, S. N., Julian, J., Hanna, K., Warner, C., Julian, R., Bandyopadhyay, R., Bennett, J. G., Bessoff, A., Branch, M., Corley, R., Dewitt, C., Eriksen, J.-D., Frommeyer, S., Gonzalez, A., Herlevich, M., Hon, D., Marin-Franch, A., Marti, J., Murphey, C., Rambold, W., Rashkin, D., Leckie, B., Gardhouse, W. R., Fletcher, M., Hardy, T., Dunn, J., and Wooff, R., "FlAMINGOS-2: the facility 
near-infrared wide-field imager and multi-object spectrograph for Gemini," in [Ground-based and Airborne Instrumentation for Astronomy II], Proceedings of the SPIE 7014, 70140V (July 2008).

[33] Kogelnik, H., "Reconstructing Response and Efficiency of Hologram Gratings," in [Modern Optics], Fox, J., ed., 605 (1967). 